\title{
Parathyroid Surgery Outcome at King Salman Armed Forces Hospital
}

\author{
Mohannad Eledreesi ${ }^{*}$, Khalid Alsubaie1, Yaser Kelany1, Yousif Alalawi², Attiya Alzahrani² \\ ${ }^{1}$ Senior Registrar General Surgery, Department of Surgery in King Salman Aramed Forces Hospital, Tabuk, Saudi Arabia \\ ${ }^{2}$ Consultant Breast and Endocrine Surgery, Department of Surgery in King Salman Aramed Forces Hospital, Tabuk, Saudi Arabia \\ Email: ^drmaledrisy@gmail.com
}

How to cite this paper: Eledreesi, M., Alsubaie, K., Kelany, Y., Alalawi, Y. and Alzahrani, A. (2022) Parathyroid Surgery Outcome at King Salman Armed Forces Hospital. Surgical Science, 13, 91-97. https://doi.org/10.4236/ss.2022.133012

Received: January 6, 2022

Accepted: March 5, 2022

Published: March 8, 2022

Copyright (c) 2022 by author(s) and Scientific Research Publishing Inc. This work is licensed under the Creative Commons Attribution International License (CC BY 4.0).

http://creativecommons.org/licenses/by/4.0/

Open Access

\section{Abstract}

Introduction: The aim of this study is to review the surgical outcome of parathyroidectomy at one institution over nine years in form of methods of diagnosis, type of surgery, postoperative complications and histopathology findings. Methods: This is a retrospective study from a single institution (King Salman North West Armed Forced Hospital (KSAFH), Tabuk, Saudi Arabia). All cases underwent parathyroidectomy from 1/1/2010 until 31/12/2018 were retrieved from the archives of the department of surgery. Data were extracted from the patients' charts. They included demographical data, presence of comorbidities, laboratory and radiological investigations, type of surgery, postoperative complications and histopathology findings. Inclusion criteria include patients older than 13 years old. Exclusion criteria include patients younger than 13 years old, hyperparathyroidism cured with medication, patients unwilling to give informed consent and patients with serious underlying medical conditions that restrict diagnostic testing or therapy such as congestive cardiac failure. Results: A total of 30 patients underwent parathyroidectomy at KSAFH. 20 out of 30 patients were female $(66.7 \%)$. The mean age was $35-45$ years old. Diabetes mellitus was found in 5 patients (16.7\%), while hypertension was seen in 10 patients (33.3\%). 6 patients (20\%) had end stage renal disease (ESRD). The data showed 24 patients with primary hyperparathyroidism (80\%), 5 patients with secondary hyperparathyroisim (16.7\%) and one patient with tertiary hyperparathyroidism (3.3\%). Our study demonstrated that 23 patients underwent partial parathyroidectomy (76.6\%), while 7 patients underwent total parathyroidectomy (23.3\%). According to the method used for diagnosis of hyperparathyroidism, blood tests were used in 27 patients $(90 \%)$, Sestamibi parathyroid scan in 26 patients (86.6\%), ultrasound scanning in 28 patients $(93.3 \%)$ and bone scan in 9 patients (30\%). Sensitivity of Sestamibi scan in primary hyperparathyroidism (91.7\%) and (66\%) for secondary hyperparathyroidism. Mean operative time was 79 minutes. Mean hospital stay was 4 
days. Postoperative ccomplications included hypocalcemia in 9 patients (30\%), wound infection was ( $0 \%)$, hematoma formation in 2 patients $(6.67 \%)$, seroma formation in 1 patient $(3.33 \%)$, vascular injury (0\%) and recurrence rate in 2 patients $(6.67 \%)$. Histopathology findings included parathyroid adenoma in 22 patients $(73.3 \%)$, parathyroid hyperplasia in 6 patients $(20 \%)$ and parathyroid carcinoma in 2 patients (6.7\%). Rate of cure was $93.3 \%$. Conclusions: Hyperparathyroidism is more common in female patients than male patients. High sensitivity for sestamibi scan in primary hyperparathyroidism. ESRD is the most common cause of secondary hyperparathyroidism. Most common complication is hypocalcemia. High rate of cure following parathyroid surgery.

\section{Keywords}

Parathyroid, Parathyroid Surgery, Hyperparathyroidism

\section{Introduction}

The main benefit of treating hyperparathyroidism with parathyroidectomy is potential to treat signs and symptoms of the disease. Primary hyperparathyroidism affects multiple systems of the body. Most patients with primary hyperparathyroidism have significant comorbidities associated with the condition. Patient can be with mild parathyroid disease or severe parathyroid disease and both are likely to benefit from parathyroid surgery. Usually there are improvements in bone disease after parathyroid surgery [1]. Hyperparathyroidism is a disease that is seen often in the United States. Patients may present with a wide variety of symptoms affecting multiple organs, but frequently they are found to be hyperparathyroid on a routine blood examination. Although, these patients may be asymptomatic, new consensus guidelines exist for when they should undergo surgery and several studies have shown multiple benefits from operative intervention [2]. The aim of this study is to describe the outcomes of parathyroidectomy in King Salman Armed Forces Hospital (KSAFH), Tabuk, Saudi Arabia.

\section{Methods}

Approval was obtained from the local ethical and research committee. This was a retrospective randomised study. Demographic data of patient collected presence of comorbidities reported and surgical complication reported. We reviewed the medical records of patients who underwent parathyroid surgery in King Salman Armed Forces Hospital, Tabuk, Saudi Arabia from 1/1/2010 till 31/7/2018 with minimum 12 - 24 months follow up. Our aim to compere between outcome of parathyroid surgery with regard to comorbidities, method of diagnosis, typer of surgery, operative time, complication rate, rate of cure, recurrence rate and histopathological finding. Our inclusion criteria were age more than 13 years and post parathyroid surgery. Exclusion criteria less than 13 years, hyperpara- 
thyroidism cured with medication, patient unwilling to give informed consent and patient with serious underlaying medical condition such as congestive cardiac disease.

\section{Operative Description}

In bilateral exploration, a transverse low collar incision is made about one finger wide above the clavicular head in an existing skin crease. The dissection is carried down through the subcutaneous tissue and the platysma muscle. Hemostasis is obtained by electrocautery or ligation using ties.

Dissection continues to elevate the upper subplatysmal flap to the thyroid notch and the lower flap to the sternal notch. Self-retaining retractors are used to retract the skin flaps. The deep fascia is opened by a midline incision along the median raphe of the strap muscles. Dissect the fascia along the anterior margins of the sternocleidomastoid muscle and either retract the strap muscles or divide the sternohyoid muscles and sternothyroid muscles vertically. Blunt dissect to the plane of cleavage between sternocleidomastoid muscle and the outer boundaries of the sternothyroid muscle. A midline vertical incision is made between the sternohyoid muscles, extending from the thyroid notch to the level of the sternal notch. The sternohyoid muscles are then elevated to develop a plane between the thyroid gland and the sternohyoid muscles. Blunt dissection with the index finger or other blunt instrument such as a Kittner is performed in this plane to the lateral edge of the thyroid to mobilize the entire lateral surface of the thyroid and expose the paratracheal space between the trachea and carotid sheath. Ligate the middle thyroid vein, then retract the thyroid lobe medially and anteriorly; the recurrent laryngeal nerve and the parathyroid glands can be identified at the upper and lower poles of the thyroid lobe. A similar exploration is made on the other side. The serum sample of PTH is collected for the baseline level. The solitary adenoma often appears to be enlarged in size and discolored. The enlarged gland is dissected and divided from the surrounding tissue without injuring the recurrent laryngeal nerve especially isolating the inferior parathyroid glands. It is important not to rupture the gland capsule in cases of malignancy but also in benign diseases to prevent parathyromatosis. The tissue specimen is sent for frozen section pathology to confirm it is the parathyroid gland. The PTH serum level is repeated, the removal of the overactive parathyroid gland is confirmed if the level drops $>50 \%$ AND to within the normal PTH range. Continue to explore additional adenoma if the PTH level doesn't fall to the expected level. Irrigate the wound, approximate the strap muscles with interrupted absorbable sutures, close the platysma with interrupted absorbable suture, close the skin with a running subcuticular absorbable suture. With preoperative localization of abnormal parathyroid gland, focused parathyroidectomy can be performed only in the imaging identified area. The incision size, the extent of dissection, and the duration of surgery are limited. 


\section{Results}

A total of 30 patient underwent parathyroidectomy at King Salman Armed Forces Hospital, of these 20 were female $(66.7 \%)$ and 10 were male $(33.3 \%)$. Mean age was 47 years. Comorbities include diabetes 5 (33.3\%), hypertension 10 (33.3\%) and end stage renal disease (ESRD) 6 (20\%). ESRD duration was less than two years $0(0 \%), 2$ - 3 years one cases, 3 - 4 years 3 cases, 4 - 5 years one case and more than 5 years one case. The type of hyperparathyroidism was 24 (80\%) primary, $5(16.7 \%)$ secondary and one $(3.3 \%)$ tertiary. Type of operation partial (PP) 23 (76.6\%) and total parathyroidectomy (TP) 7 (23.3\%) (Figure 1 \& Figure 2).

With method used for diagnosis blood test 27 (90\%), Sestamibi parathyroid scan $26(86.6 \%)$, ultrasound 28 (93.3\%) and bone scan 9 (30\%). Mean operative time was 61 minute, 43 minutes in PP and 79 minutes in TP. Mean hospital stay 3 - 5 days. Complication rate shown hypocalcemia 9 (30\%), wound infection 0 $(0 \%)$, hematoma $2(6.67 \%)$, the two cases were in TP and no cases in PP, seroma $0(0 \%)$, vascular injury $0(0 \%)$ and recurrence rate $2(6.67 \%)$ one case in PP and one case in TP. Histopathological finding of parathyroid adenoma 22 (73.3\%), hyperplasia 6 (20\%) and parathyroid carcinoma 2 (6.7\%). Largest size was 9 CM. Rate of cure $94 \%$ (Table 1, Figure 3 \& Figure 4 ).

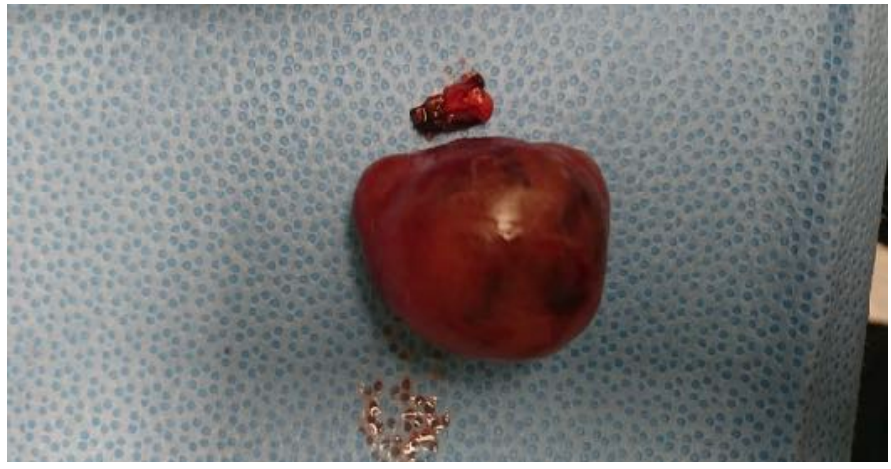

Figure 1. Single Parathyroidectomy.

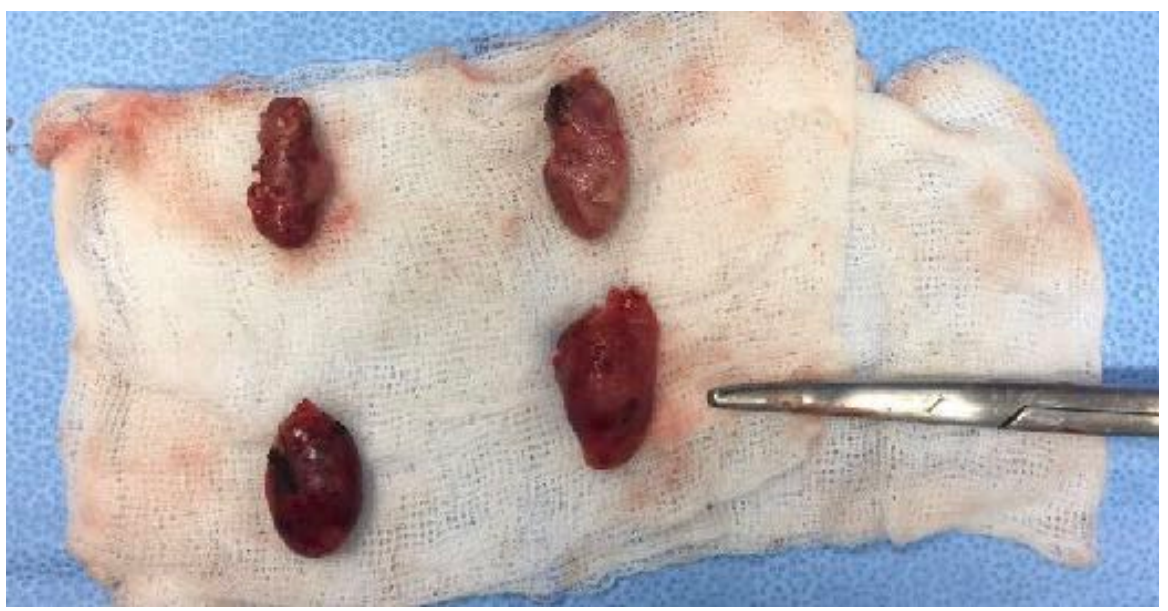

Figure 2. Total Parathyroidectomy. 
Table 1. Parathyroid surgery outcome: compartion between Partial Parathyroidectomy (PP) and Total Parathyroidectomy (TP).

\begin{tabular}{ccc}
\hline Type of operation & PP & TP \\
\hline No. of cases & 23 & 7 \\
Time (minute) & 43 & 79 \\
Recurrence & 1 & 1 \\
Wound infection & 0 & 0 \\
Seroma & 0 & 0 \\
Hematoma & 0 & 2 \\
vascular injury & 0 & 0 \\
\hline
\end{tabular}

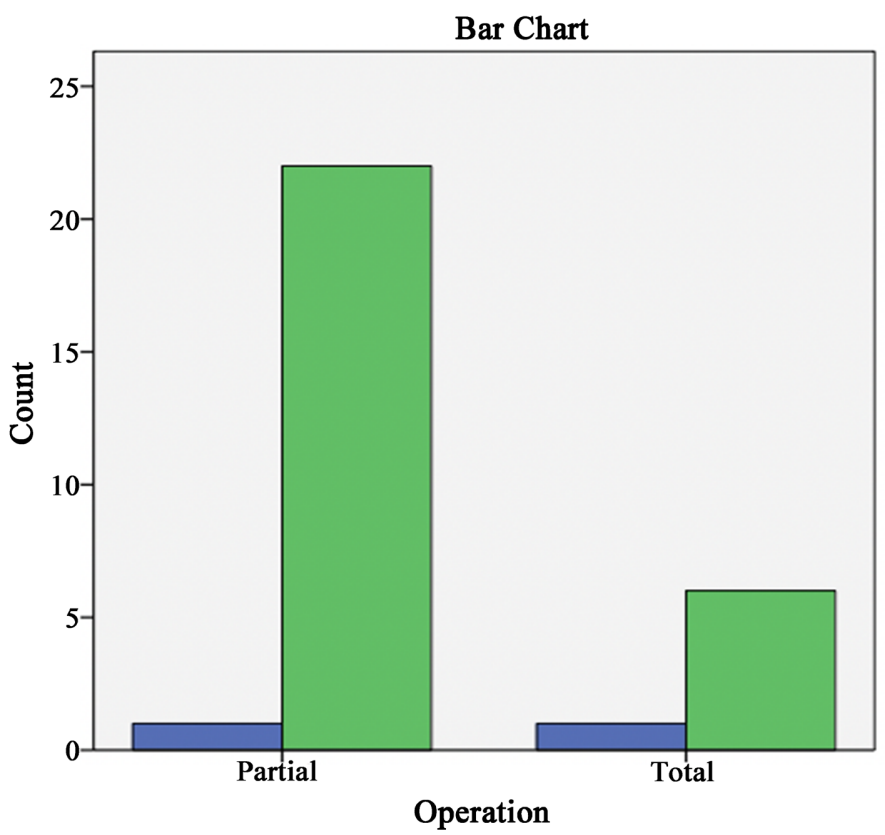

Recurrence

ㅁ. Yes

$\square$ No

Figure 3. Recurrence rate with type of operation.

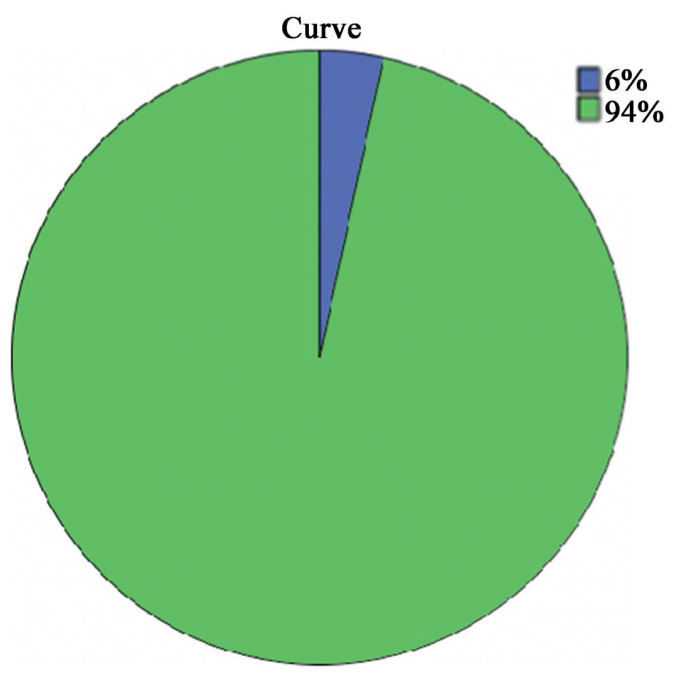

Figure 4. Cure rate. 


\section{Discussion}

Parathyroidectomy is the definitive therapy for primary hyperparathyroidism but may also be necessary in secondary or tertiary hyperparathyroidism. Bilateral neck exploration is the standard operation, especially optimal for patients who have multiple gland disease or non-localizing preoperative imaging studies. With improved preoperative localization techniques and intraoperative PTH monitoring, minimally invasive parathyroidectomy is widely used in unilateral exploration. Even a four-gland exploration can be performed in a minimally invasive fashion [5]. Surgery of primary hyperparathyroidism is the only curative therapy. Cure rate for primary disease is about $(94 \%-97 \%)$ with complication rate about $(1 \%-2 \%)$. A study by Sosa et al. demonstrated that surgeon experience determined the length of hospital stay and rate of complication. If undertaken by an experienced endocrine surgeon, minimal invasive parathyroidectomy is a best operation if preoperative study localizes an adenoma, otherwise bilateral neck exploration is undertaken. Decrease in kidney stone after parathyroid surgery is documented. About $70 \%$ of people experience improvements in health related quality of life after parathyroid surgery. Improvements in surgical technique have made parathyroid surgery a better option for many people worldwide. Primary hyperparathyroidism disproportionately affects older patients, who may face higher thresholds for surgical intervention compared to young patients [3]. Silverberg SJ, Shane E, Jacobs TP, Siris E, and Bilezikian JP result show primary hyperparathyroidism, parathyroidectomy results in the normalization of biochemical values and increased bone mineral density. Most asymptomatic patients who did not undergo surgery did not have progression of disease, but approximately one quarter of them did have some progression [4]. Drawbacks of our study were single center, nonrandomized, biased selection, small size and short follow up.

\section{Conclusion}

Hyperparathyroidism is more common in female patients than male patients. High sensitivity for sestamibi scan in primary hyperparathyroidism. ESRD is the most common cause of secondary hyperparathyroidism. Most common complication is hypocalcemia. High rate of cure following parathyroid surgery.

\section{Acknowledgments}

We acknowledge the support of academic affairs department at King Salman Armed Forces Hospital, Tabuk, KSA. Disclosures: none

\section{Conflicts of Interest}

The authors declare no conflicts of interest regarding the publication of this paper.

\section{References}

[1] Chan, A.K., Duh, Q.Y., Katz, M.H., Siperstein, A.E. and Clark, O.H. (1995) Clinical 
Manifestations of Primary Hyperparathyroidism before and after Parathyroidectomy. A Case-Control Study. Annals of Surgery, 222, 402-412.

https://pubmed.ncbi.nlm.nih.gov/7677469/

https://doi.org/10.1097/00000658-199509000-00017

[2] Zarebczan, B. and Chen, H. (2011) Influence of Surgical Volume on Operative Failures for Hyperparathyroidism. Advances in Surgery, 45, 237-248.

https://www.ncbi.nlm.nih.gov/pmc/articles/PMC3224988/

https://doi.org/10.1016/j.yasu.2011.03.003

[3] Wu, B., Haigh, P.I., Hwang, R., Ituarte, P.H.G., Liu, I.-L.A., Hahn, T.J. and Yeh, M.W. (2010) Underutilization of Parathyroidectomy in Elderly Patients with Primary Hyperparathyroidism. The Journal of Clinical Endocrinology \& Metabolism, 95, 4324-4330. https://doi.org/10.1210/jc.2009-2819 https://www.ncbi.nlm.nih.gov/pmc/articles/PMC2936062/

[4] Silverberg, S.J., Shane, E., Jacobs, T.P., Siris, E. and Bilezikian, J.P. (1999) A 10-Year Prospective Study of Primary Hyperparathyroidism with or without Parathyroid Surgery. The New England Journal of Medicine, 341, 1249-1255.

https://pubmed.ncbi.nlm.nih.gov/10528034/ https://doi.org/10.1056/NEJM199910213411701

[5] Sosa, J.A. and Udelsman, R. (2003) Minimally Invasive Parathyroidectomy. Surgical Oncology, 12, 125-134. https://pubmed.ncbi.nlm.nih.gov/12946483/

https://doi.org/10.1016/S0960-7404(03)00041-0 